Unfallchirurg 2016 $119: 706-707$

DOI 10.1007/s00113-016-0220-2

Online publiziert: 28. Juli 2016

(c) Springer-Verlag Berlin Heidelberg 2016

CrossMark

\section{Stengel ${ }^{\prime} \cdot$ C. Bartl ${ }^{2}$}

'Zentrum für Klinische Forschung, Unfallkrankenhaus Berlin, Berlin, Deutschland

${ }^{2}$ ZOOOM, Zentrum für Orthopädie, Osteoporose, Osteopathie - München, München, Deutschland

\title{
Distale Radiusfrakturen - Evidence is Shlevidence
}

Für Interessierte an Hintergründen und Grundprinzipien evidenzbasierter Medizin war in den frühen 2000er-Jahren das Centre for Evidence-Based Medicine (CEBM) am Radcliff Hospital in Oxford praktisch eine Pilgerstätte. Deutsche Besucher traten die Reise dorthin häufig mit völlig falschen Vorstellungen an. Erwarteten sie eine Evidenzkathedrale mit strengem Glauben daran, dass ohne wissenschaftliche Daten (im Idealfall aus randomisierten Studien) keine Medizin mehr praktiziert werden könnte, gingen die britischen Kolleginnen und Kollegen seit jeher deutlich nüchterner mit der Verknüpfung ärztlicher Fähigkeiten und Kenntnissen des wissenschaftlichen Status quo um. Bei einem gemeinsamen Grillabend prägte der damalige Direktor des CEBM, Dr. Martin Dawes, mit Augenzwinkern den Kunstbegriff „Evidence is Shlevidence“. Die Botschaft an die Teilnehmer vom Kontinent war simpel - macht aus der EbM-Philosophie bitte kein starres Dogma, lasst ihr Luft zum Atmen und erkennt ihre Dynamik.

In der Rückschau bedurfte es wohl dem besten aus beiden Welten, um das Gesundheitswesen wissensbasierter und transparenter zu machen: der deutschen Tendenz $\mathrm{zu}$ formalisieren und $\mathrm{zu}$ zertifizieren und dem angloamerikanischen Pragmatismus. Frei nach Jürgen Windeler sollte EbM ein Instrument sein, um zwischen Therapiefreiheit und Beliebigkeit in der Medizin zu unterscheiden (und letztere $\mathrm{zu}$ eliminieren).

Dies gilt für alle Fachgebiete und Versorgungsebenen. In Unfallchirurgie und Orthopädie stand die methodische Qualität publizierter klinischer Studien in den vergangenen Jahren auf dem Prüfstand und der Trend ist ermutigend [1-6].
Dies liegt u.a. daran, dass sich praktisch alle bedeutsamen Publikationsorgane den vom International Committee of Medical Journal Editors (ICMJE) empfohlenen Standards wie prospektive Studienregistrierung, Protokollveröffentlichung, Reporting im Einklang mit CONSORT, STARD, STROBE, PRISMA etc. im Einklang mit ICH-GCP verpflichtet haben (s. u.a. http://www.equatornetwork.org). Auch besteht eine Chance für eine Forschungsförderung mit öffentlichen Mitteln nur dann, wenn ein exzellentes methodisches Gerüst konstruiert wurde. Methodische Standards sind längst in Orthopädie und Unfallchirurgie angekommen.

Dieses Heft legt den Fokus auf die distale Radiusfraktur und spannt den Bogen von der Biomechanik über die Ergebnisse vergleichender Interventionsstudien hin zu Komplikationen wie dem CRPS.

Es existieren einige methodisch exzellente klinische Studien zur Therapie dieser häufigen Verletzung. An dieser Stelle soll noch einmal, BREXIT hin oder her, der Blick auf Großbritannien gerichtet werden, dessen Gesundheitssystem an verschiedenen Stellen bemängelt wird und sicher auch nicht mit dem bundesdeutschen vergleichbar ist. Bei der Generierung valider Daten für die klinische Versorgung haben die dortigen Kolleginnen und Kollegen jedoch ein Manko abgestellt, welches bei uns noch vielfach zu beobachten ist - die Zurückhaltung in der Beteiligung an multizentrischen, klinisch-experimentellen Prüfungen (obwohl diese z. B. TraumaRegister $\mathrm{DGU}^{\circledR}$ seit Jahren vorbildlich funktioniert).

- Abb. 1 illustriert die Rekrutierungsraten der britischen DRAFFT-Studie (Distal Radius Acute Fracture Fixation Trial) [7], welche durch das Health Technology Assessment Scheme des National Institute of Health Research [HTA08/116/97] finanziert und durch ein umfangreiches methodisches, administratives und klinisch-logistisches Netzwerk unterstützt wurde. DRAFFT verglich die volare winkelstabile Plat-

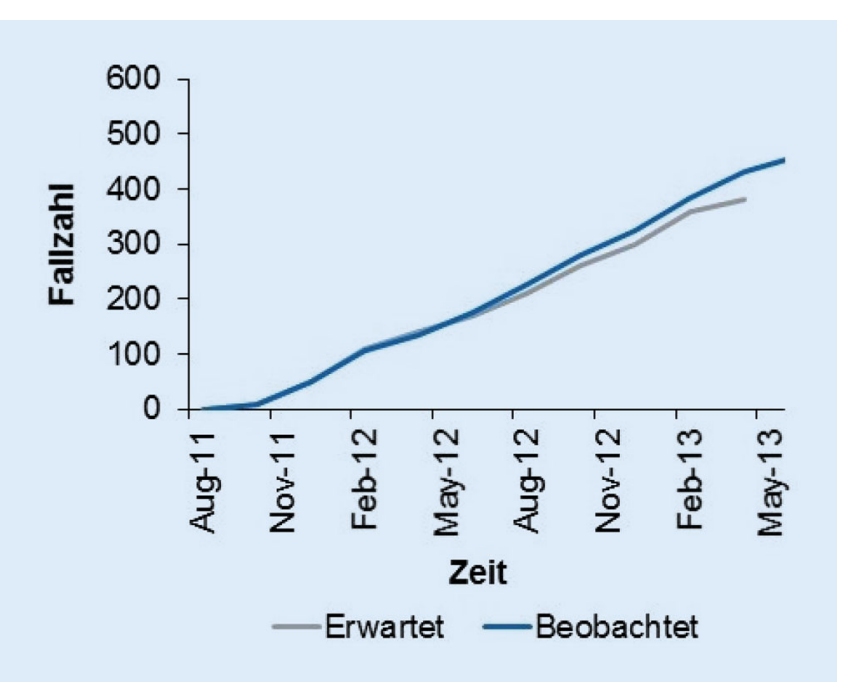

Abb. $1<$ Rekrutierungsraten des DRAFFT-Trial [7]. Beachten Sie die höher als erwartete Rekrutierungsdichte an den 18 britischen Zentren, welche dazu führte, dass in 18 Monaten statt der geplanten 390 Patienten 461 rekrutiert wurden 
tenosteosynthese mit einer perkutanen K-Draht-Fixation in einer gemischten Patientenstichprobe. Bemerkenswert war eine höher als erwartete Rekrutierungsdichte an den beteiligten 18 Zentren. Die Steuerungsgruppe entschloss sich aufgrund der einmaligen Chance, die Rekrutierung nicht bei den geplanten 390 Patienten zu stoppen, sondern den vollen Förderzeitraum auszuschöpfen und somit 461 Patienten einzuschließen.

Eine engere Zusammenarbeit zwischen universitären und nichtuniversitären unfallchirurgisch-orthopädischen Zentren ist essenziell, wenn es (neben der Registerforschung) um prospektive Studien, ob randomisiert oder nicht randomisiert, ob konfirmatorischer oder explorativer Natur, geht. In der Zukunft erwarten uns spannende Förderprogramme und Entwicklungen in der klinischen Studienmethodik, in die wir uns gemeinsam einbringen und von denen wir alle profitieren können. Evidenzbasierte Unfallchirurgie und Orthopädie ist real und facettenreich. In diesem Sinne bedanken wir uns als Gastherausgeber für die Beiträge unserer Kolleginnen und Kollegen und wünschen Ihnen viel Vergnügen und Erkenntnisgewinn bei der Lektüre.
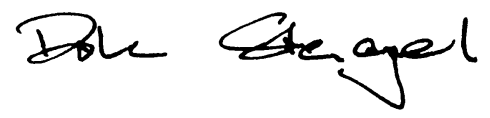

Prof. Dr. Dirk Stengel, MSc (Epi)

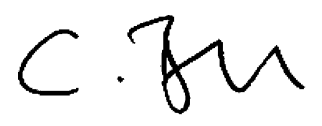

Priv.-Doz. Dr. Christoph Bartl

\section{Korrespondenzadresse}

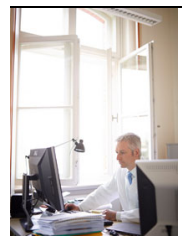

Prof. Dr. D. Stengel

Zentrum für Klinische Forschung, Unfallkrankenhaus Berlin

Warener Str. 7, 12683 Berlin,

Deutschland

dirk.stengel@ukb.de

Interessenkonflikt. D. Stengel und C. Bartl geben an, dass kein Interessenkonflikt besteht.

\section{Literatur}

1. Chess LE, Gagnier J (2013) Risk of bias of randomized controlled trials published in orthopaedic journals. BMC Med Res Methodol 13:76

2. Dijkman BG, Abouali JA, Kooistra BW et al (2010) Twenty years of meta-analyses in orthopaedic surgery: has quality kept up with quantity? J Bone Joint Surg Am 92:48-57

3. Mundi R, Chaudhry H, Mundi S, Godin K, Bhandari $M$ (2014) Design and execution of clinical trials in orthopaedic surgery. Bone Joint Res 3:161-168

4. Mundi R, Chaudhry H, Sharma R, Schemitsch E, Bhandari $M$ (2007) What is the quality of the orthopaedic literature? J Long Term Eff Med Implants 17:103-109

5. Poolman RW, Struijs PA, Krips R, Sierevelt IN, Lutz KH, Bhandari M (2006) Does a "Level I Evidence" rating imply high quality of reporting in orthopaedic randomised controlled trials? BMC Med Res Methodol 6:44

6. Rongen JJ, Hannink G (2016) Comparison of registered and published primary outcomes in randomized controlled trials of orthopaedic surgical interventions. J Bone Joint Surg Am 98:403-409

7. Costa ML, Achten J, Parsons NR et al (2014) Percutaneous fixation with Kirschner wires versus volar locking plate fixation in adults with dorsally displaced fracture of distal radius: randomised controlled trial. BMJ 349:94807

\section{Junge Ärzte legen Wert auf Work-Life-Balance}

Selbstbewusst, fordernd, ergebnisorientiert: So wird die "Generation Y" charakterisiert. Wie sehen um das Jahr 1980 geborene Mediziner ihre berufliche Perspektive? Hierzu führte die Landesärztekammer Hessen eine qualitative Studie mit 30 Jungmedizinern (20 in Weiterbildung und 10 Fachärzte) durch. In den Interviews zeigt sich, dass insbesondere die Work-Life-Balance als wichtig erachtet wird. Die Befragten wünschen sich vor allem flexible Arbeitszeiten und die Möglichkeit der Vereinbarkeit von Privatleben, Familie und Beruf. Ein wichtiger Faktor ist hier auch die Feminisierung der Medizin. Um sich vor Überlastung zu schützen, würden die jungen Ärzte persönliche Grenzen ziehen. Überstunden und eine „Aufopferung" für die Karriere würden nicht mehr ohne weiteres akzeptiert. Schließlich könne nur volle Leistung bringen, wer ausgeglichen sei, so die Argumentation. Damit wird auch der Spaß an der Arbeit assoziiert. Fast allen Befragten ist es wichtig, Aufgaben und Ziele im Rahmen ihrer Weiterbildung klar definiert zu bekommen. Dazu gehört für sie regelmäßiges Feedback und die Einhaltung von Versprechen bzw. Vereinbarungen. Idealerweise sollte die Zusammenarbeit auf „Augenhöhe" erfolgen. Strenge Hierarchien werden nicht mehr akzeptiert. Aufgrund des guten Arbeitsmarktes können es sich Ärzte in Weiterbildung leisten, Forderungen zu stellen, meinen die Befragten. Dabei wurde auch deutlich, dass sich Kollegen der älteren Generation über das sehr selbstbewusste Verhalten verwundert zeigen.

Quelle: Landesärztekammer Hessen, www.laekh.de 